\title{
Design and Thinning of Linear and Planar Antenna Arrays Using a Binary Teaching Learning Optimizer
}

\author{
A. ReCiOui* \\ Laboratory of Signals and Systems, Institute of Electrical Engineering and Electronics, \\ University M'hamed Bougara of Boumerdés, Algeria
}

\begin{abstract}
In this paper, a binary variant of the teaching learning optimization technique is used to the design and thinning of linear and planar arrays. The purpose of the optimization task is to enhance the ratio directivity/sidelobe level which turns out to be having two conflicting parameters. The binary variant of the teaching learning optimization technique searches the way of exciting some selected elements. The array thinning problem requires some elements to be excited with the others having no current in them. This is a binary (ON-OFF) problem that requires an optimization technique that can handle the binary variables. The teaching learning optimization has been proposed initially to handle real valued variables. The results show good agreement between the desired and calculated radiation patterns with reduction in resource usage in terms of power consumption.
\end{abstract}

DOI: 10.12693 /APhysPolA.130.7

PACS/topics: 84.40.Ba, 02.60.Pn, 84.40.Ua

\section{Introduction}

In the problem of array thinning, the number the possible combinations is large and it increases exponentially with the number of array elements. One needs a faster and reliable method to find the optimum solution. Nongradient based optimization methods such as genetic algorithms (GA) [1,2] and particle swarm optimization (PSO) [3] are introduced in array thinning and have proved to be useful. Haupt [4] used GA in process of thinning a linear array of 200 elements, and resulting sidelobe levels were lower than $-18 \mathrm{~dB}$ in all cases shown. Gies and Rahmat-Samii [5] used GA to thin a 40 elements linear array and achieved sidelobe levels lower than $-20 \mathrm{~dB}$. Mahanti et al. [6] used GA to thin a large linear array of uniformly excited isotropic elements to yield SLL equal to or below a fixed level, while the percentage of thinning is equal to or above a fixed value. Clonal selection $[7,8]$ and bees algorithm [9] are two other optimization tools which are used recently in different problems of array synthesis field with successful results.

The teaching learning based optimization (TLBO) technique was proposed recently by Rao et al. $[10,11]$. The TLBO exists for real valued problems. A binary teaching learning based optimization (BTLBO) based method for finding the best element excitation combination is proposed here.

The objective of this paper is to find a configuration for a thinned array which has a normalized sidelobe level (SLL) as low as possible and with a half-power beamwidth (HPBW) not so much more than the case of the uniform array which has the narrowest possible HPBW. This goal is achieved by using BTLBO as the

*e-mail: recmad2006@yahoo.fr optimization tool. The results are excellent for arrays of 50 elements as compared to the uniform case.

\section{Problem formulation}

For a linear array, the far field array factor can be written as [1]:

$$
\mathrm{AF}_{\mathrm{a}}(\theta)=2 \sum_{k=1}^{N} a_{k} \cos \left(\frac{2 \pi}{\lambda} x_{k}\left(\sin (\theta)-\sin \left(\theta_{0}\right)\right)\right),
$$

where $\lambda$ is the wavelength, $\theta$ is the scanning angle from broadside, $\theta$ is the direction of the main beam $\left(0^{\circ}\right.$ for broadside), $a_{k}$ is the amplitude of the $k$-th element and $x_{k}$ is the position of the $k$-th element with respect to the array center.

For the two-dimensional array with $M \times N$ elements, the array factor is given as

$$
\operatorname{AF}(\theta, \phi)=\sum_{m=1}^{M} \sum_{n=1}^{N_{M}} I_{n m} \exp \left(\mathrm{i} k \sin (\theta) \cos \left(\phi-\phi_{n m}\right)\right) .
$$

The array factor here is evaluated over the planes $\phi=0^{\circ}$ and $\phi=90^{\circ}$ to make the elevation angle $\theta$ as the variable over which the array characteristics are evaluated.

The array factor has to be normalized with respect to the highest value. The directivity is maximized indirectly in relation to the minimization of the half power beamwidth as

$$
F_{1}=\theta_{2}-\theta_{1},
$$

where $\theta_{1}$ and $\theta_{2}$ are the angles corresponding to $\frac{\max (\mathrm{AF})}{\sqrt{2}}$.

To achieve the lowest peak SLL, the second term in the optimization fitness function is chosen to be merely the sidelobe level. Then, the fitness function to minimize is

$$
f=F_{1}+F_{2} \text {. }
$$

\section{The teaching learning based optimization}

TLBO is population based method. In this optimization algorithm, a group of learners is considered as population and different design variables are considered as 
different subjects offered to the learners and learners result is analogous to the "fitness" value of the optimization problem. In the entire population, the best solution is considered as the teacher. The working of TLBO is divided into two parts: "Teacher phase" and "Learner phase", which are explained below:

Teacher phase: in this phase the best student is chosen from the population (the class) according to the fitness function and set as a teacher. Since the teacher is the highest learned person in the class, he puts effort to disseminate knowledge among students, so that he tries to bring the mean level of the class up to his level, the new mean of the class depends on two things:

- The ability of the teacher i.e. his method in teaching is good or bad and this is represented by a factor $t_{\mathrm{f}}$ called "teaching factor", it can be 1 or 2 (those values are concluded from experiments).

- The ability of the student to receive and understand concepts from his teacher.

Learner phase: as known, when a student does not understand his teacher or he wants to have more knowledge, he will interact with one of their fellow students. If he finds his friend better than himself he will learn from him otherwise he will not.

\section{Results and discussions}

\subsection{Linear array optimization}

A 50-element linear array is optimized for sidelobe level and directivity. The elements are uniformly separated at $\frac{\lambda}{2}$ and also equally phased. The algorithm results in an array of 36 turned-on elements which has a SLL of $-21.11 \mathrm{~dB}$ as compared to $-12.71 \mathrm{~dB}$ for the uniformly excited array. This represents $66 \%$ reduction in sidelobe level with power consumption reduced to $72 \%$ the power used in the uniform case. However, this has been achieve with a penalty paid in the increase in the HPBW (hence a less directive array) by $15 \%$ from $4^{\circ}$ to $4.6^{\circ}$.

\subsection{Planar array optimization}

The planar array factor being a product of two orthogonal array factors is evaluated as a product of two array factors in the planes $\phi=0^{\circ}$ and $\phi=90^{\circ}$. The number of elements in each dimension is taken to be 50 which make the total array size being 2500 elements.

\subsubsection{In the plane $\phi=0^{\circ}$}

The optimized array factor exhibits a sidelobe level of $-20.06 \mathrm{~dB}$ which is better by $58 \%$ compared to the uniformly fully excited array. The percentage use of elements is $80 \%$ meaning 40 elements are turned on. The directivity increase (HPBW) here is at around $12.5 \%$ as the $\mathrm{HPBW}$ increased from $4^{\circ}$ to around $4.5^{\circ}$.

\subsubsection{In the plane $\phi=90^{\circ}$}

The BTLBO could achieve a good result in this plane as the sidelobe level is now at $-23.02 \mathrm{~dB}$ which represents an improvement of $80 \%$ compared to the uniform case. The number of turned on elements is now at 42 which constitutes $16 \%$ reduction in power consumption. The directivity (precisely HPBW) is increased by $20 \%$ from $4^{\circ}$ to $4.8^{\circ}$.

Overall, for the linear array optimization task, though a loss in directivity is noticed, the gain in sidelobe level is dominating. Indeed, the ratio directivity (HPBW)/sidelobe level is better than the uniform case. As for the planar case, the overall sidelobe level (twodimensional) is at worst equal to -20.06 which is a suitable value for most nowadays communication systems. Regarding directivity, the overall directivity being proportional to the product of the individual HPBWs, it is expected to remain within a relatively reasonable value compared to the uniform case. The number of elements that are turned on is $42 \times 40=1680$ which represents a reduction in power consumption of $32.8 \%$ compared to when all the 2500 elements are excited.

\section{Conclusions}

The problem of one and two-dimensional array thinning using TLBO has been addressed in this paper. This problem aims finding the number of elements to be excited to reduce power consumption while preserving the array characteristics at adequate levels. In literature, the existing TLBO version treats only real valued problems. In this work, we have proposed a binary version utilizing the same mechanism of the real valued TLBO. The results reveal an enhancement in the array performance in terms of the compromise directivity/sidelobe level with reduction in power consumption that reached $30 \%$ in the case of planar arrays.

\section{References}

[1] A. Recioui, A. Azrar, Microw. Opt. Technol. Lett. 49, 1619 (2007).

[2] A. Recioui, A. Azrar, H. Bentarzi, M. Dehmas, M. Chalal, Int. J. Microw. Opt. Technol. 3, 524 (2008).

[3] M.M. Khdier, C.G. Christodoulou, IEEE Trans. Antennas Propag. 53, 2674 (2005)

[4] R.L. Haupt, IEEE AP-S Trans. 42, 993 (1994).

[5] D. Gies, Y. Rahmat-Samii, Microwave Opt. Technol. Lett. 38, 168 (2003).

[6] G.K. Mahanti, N. Pathak, P. Mahanti, Prog. Electromagn. Res. (PIER) 75, 319 (2007).

[7] B. Babayigit, A. Akdagli, K. Guney, J. Electromagn. Waves Appl. 20, 1007 (2006).

[8] A. Akdagli, K. Guney, B. Babayigit, J. Electromagn. Waves Appl. 21, 215 (2007).

[9] K. Guney, M. Onay, Prog. Electromagn. Res. (PIER) 70, 21 (2007).

[10] R.V. Rao, V.J. Savsani, D.P. Vakharia, ComputerAided Des. 43, 303 (2011)

[11] R.V. Rao, V.J. Savsani, D.P. Vakharia, Inform. Sci. 183, 1 (2012). 